\title{
Light Propagation Characteristics of Straight Single-Line-Defect Waveguides in Photonic Crystal Slabs Fabricated Into a Silicon-on-Insulator Substrate
}

\author{
Toshihiko Baba, Member, IEEE, Ayumu Motegi, Takeshi Iwai, Naoyuki Fukaya, Yoshitaka Watanabe, and \\ Atsushi Sakai
}

\begin{abstract}
Straight single-line defect optical waveguides in photonic crystal slabs are designed by the finite difference time-domain method and fabricated into a silicon-on-insulator (SOI) wafer. By employing an airbridge structure, clear light propagation for both polarizations is observed without any leakage along the waveguide. This experimental result is well explained by photonic bands of pure guided modes. Minimum propagation loss is estimated to be $11 \mathrm{~dB} / \mathrm{mm}$. This value is lower than that reported so far for threeline-defect waveguides with an SOI slab structure and almost comparable to that for an index confinement waveguide with a rectangular Si core. This propagation loss is dominated by the scattering loss by some irregularities. However, photonic crystal waveguides have the possibility of an essential lower scattering loss than in the index confinement waveguide because of the inhibition of radiation modes by the photonic bandgap.
\end{abstract}

Index Terms-FDTD, photonic bandgap, photonic crystal, SOI, waveguide.

\section{INTRODUCTION}

$\mathbf{T}$ HE PHOTONIC crystal (PC) slab is a high refractive index slab with low index claddings and two-dimensional (2-D) periodic airholes. In recentyears, an optical waveguide composed of a line defect in such PC slab has become one of the topics in this research field. There are two reasons for this. One is the potential that the photonic bandgap (PBG) of the PC slab allows sharp bends, branches, and other components, which are

Manuscript received August 31, 2001; revised November 27, 2001. This work was supported in part by the Ministry of Education, Science, Sports and Culture under Grant-in-Aid 10210203 and by CREST 530-13 of Japan Science and Technology Corporation.

T. Baba and A. Motegi are with the Department of Electrical and Computer Engineering, Yokohama National University, Yokohama 240-8501, Japan (e-mail baba@dnj.ynu.ac.jp).

T. Iwai was with the Department of Electrical and Computer Engineering Yokohama National University, Yokohama 240-8501, Japan. He is now with NEC Corporation, Minatoku, Tokyo 108-8001, Japan.

N. Fukaya was with the Department of Electrical and Computer Engineering, Yokohama National University, Yokohama 240-8501, Japan. He is now with Furukawa Electric Inc., Yokohama Laboratories, Nishiku, Yokohama 220-0073, Japan

Y. Watanabe was with the Department of Electrical and Computer Engineering, Yokohama National University, Yokohama 240-8501, Japan. He is now with Toyota, Inc., Aichi 471-8571, Japan.

A. Sakai was with the Department of Electrical and Computer Engineering, Yokohama National University, Yokohama 240-8501, Japan. He is now with Ricoh Company, Ltd., Tsuzukiku, Yokohama 224-0035, Japan.

Publisher Item Identifier S 0018-9197(02)05686-5. useful for constructing a large-scale functional photonic chip. The other is the relatively easy fabrication process, in which only the thin slab is etched to form the PC slab and the waveguide. However, few reports have demonstrated the clear correspondence between theoretical and experimental light propagation characteristics. It is thought to be due to complex photonic bands, which require careful design of such waveguides.

The line defect waveguide was first discussed by Meade et al. [1]. Then, a sharp bend was theoretically calculated by Mekis et al. [2] and some other components by Yonekura et al. [3]. These works assumed 2-D PCs composed of dielectric rods and the air background. This type of PC provides a wide PBG for the polarization normal to the 2-D plane (we call it TM polarization in the following). The line defect waveguide in this PC allows a wide design window and a wide transmission band. However, for experimental demonstrations, some optical confinement is necessary in the third dimension. This optical confinement is difficult to realize in this type of PC, since the waveguide core is composed of air. On the other hand, the line defect waveguide in the PC slab has a dielectric core and realizes the third optical confinement by the total internal reflection. The concept of the PC slab originates from a one-dimensional (1-D) scheme by Foresi et al. [4] but is applicable to 2-D schemes. In particular, the PC slab with triangular lattice airholes exhibits a wide PBG for the polarization parallel to the 2-D plane (we call it TE polarization in the following). As the first experiment, we introduced line defects of missing airholes in a PC slab as a waveguide channel [5], [6]. We used the bonding process of a GaInAsP epitaxial film on a host substrate to form the PC slab and confirmed the light propagation at $1.5-\mu \mathrm{m}$-wavelength range from the scattered light along the waveguide. Lin et al., demonstrated another type of line defect waveguide, i.e., a waveguide composed of airholes with a detuned diameter [7]. In both experiments, however, waveguides were too short ( $\leq 25 \mu \mathrm{m}$ in length) to evaluate the propagation loss. Tokushima et al. [8] and Lončar et al. [9] simplified the fabrication process by using a silicon-on-insulator (SOI) wafer, as reported for the 1-D PC [4]. They fabricated relatively long waveguides of missing airholes, but the light output was too weak to evaluate losses quantitatively. We fabricated three-line-defect waveguides of missing airholes into an SOI wafer and measured a propagation loss of $60 \mathrm{~cm}^{-1}$ $(26 \mathrm{~dB} / \mathrm{mm})$ by using the Fabry-Perot resonance method [10]. 


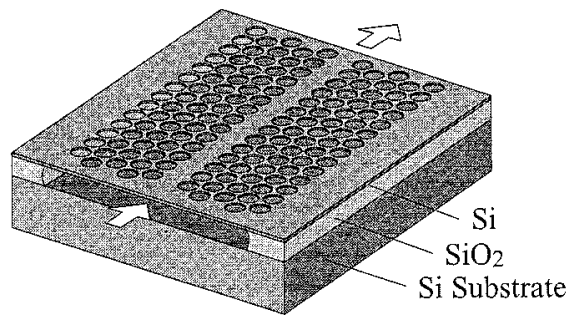

(a)

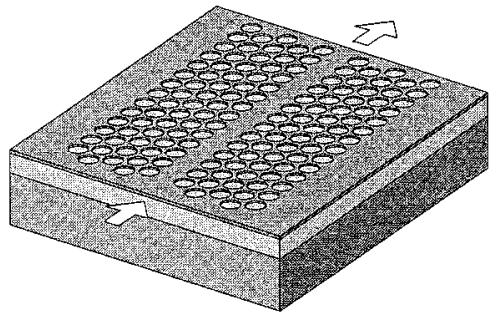

(b)

Fig. 1. Schematics of PC waveguides. (a) Airbridge type and (b) SOI slab type.

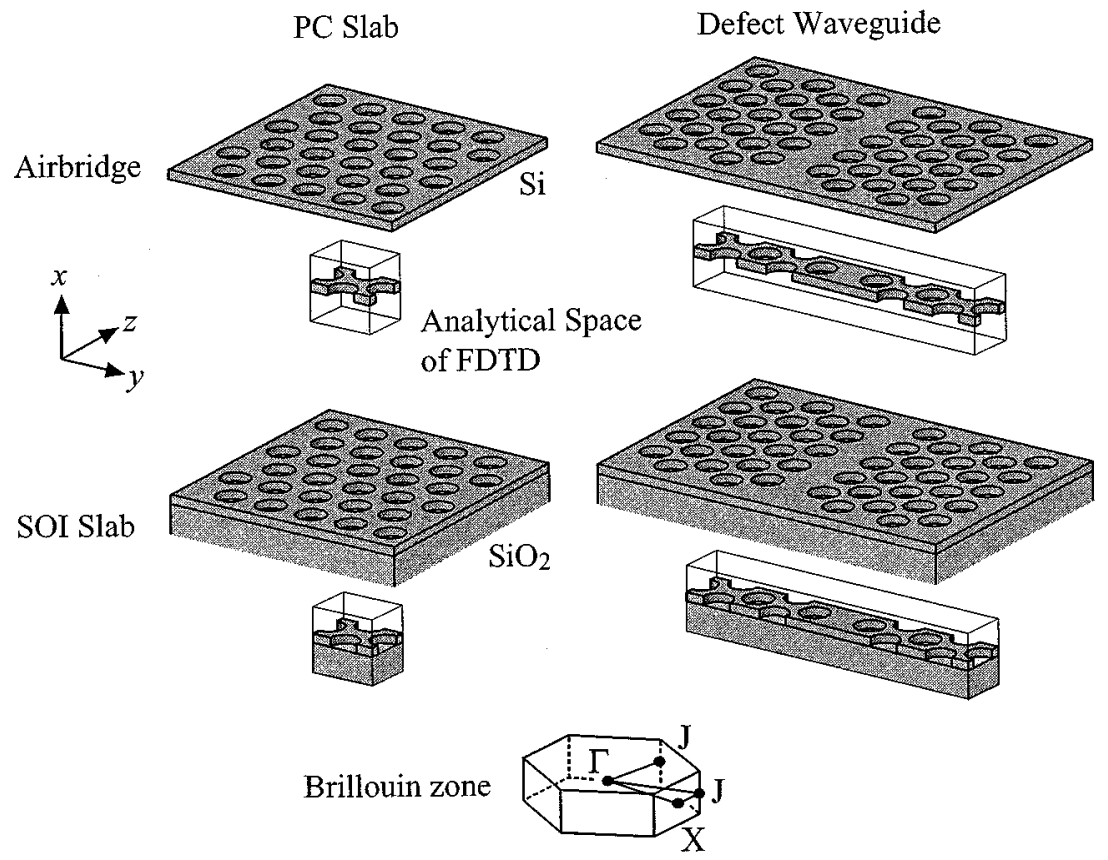

Fig. 2. FDTD calculation models of PC slabs and PC waveguides with the coordinate system and the Brillouin zone

Smith et al. reported a similar value of $50 \mathrm{~cm}^{-1}(22 \mathrm{~dB} / \mathrm{mm})$ for a three-line-defect waveguide in an (In)GaAs-AlGaAs PC slab, which was evaluated by measuring the in-plane propagation of photoluminescence [11]. These results suggest that line defect waveguides in PC slabs require more careful design than that for a waveguide in the dielectric rod 2-D PC.

As the theoretical work on PC slabs, Johnson et al.explained the relation of photonic bands and the light cone and the mixing of polarizations in an asymmetric PC slab [12]. Chutinan et al. [13], Johnson et al. [14], and Lončar et al. [15] reported photonic bands of single-line-defect waveguides of missing airholes in a PC slab. Their calculations indicated the importance of air claddings, i.e., airbridge structure, to avoid leakage conditions of guided modes, i.e., light cone and slab modes, and showed some designs to obtain pure guided modes. Notomi et al. [16] indicated the importance of a large slope of band curve. It corresponds to a large group velocity $c / n_{g}$ for the vacuum velocity of light $c$ and the group index $n_{g}$, which provides a practical low propagation loss. They discussed a modification of the line defect to expand the design flexibility.

Based on these works, the purpose of this study is to confirm the correspondence between theoretical and experimental light propagation characteristics in straight single-line-defect waveguides of missing airholes in PC slabs. In the following, we simply call this type of waveguide a $\mathrm{PC}$ waveguide. We investigated two types of waveguides with different PC slabs, as shown in Fig. 1. One is the airbridge type with air claddings and the other is the SOI slab type with air and $\mathrm{SiO}_{2}$ claddings. In Section II, we describe the photonic band calculation by the three-dimensional (3-D) finite difference time-domain (FDTD) method. Compared with the plane-wave expansion (PWE) method, advantages of this method are the faster convergence for complex structures of PC waveguides and the feasibility of investigating photonic bands both below and above the light line within a realistic calculation time. We discuss general properties and the optimum design of photonic bands of pure guided modes with a large slope in PC waveguides. In Section III, we describe the detail of our fabrication process and show typical irregularities in fabricated waveguides. In Section IV, we first explain a method for estimating the propagation loss and then show various light propagation characteristics. We discuss the correspondence between the theory and the experiment. Finally, in Section V, we discuss the scattering loss by the irregularities as the origin of the propagation loss. Showing the calculation of the scattering loss in an index confinement waveguide, we discuss the possibility of an essential lower loss in PC waveguides than in index confinement waveguides. 


\section{FDTD PHOTONIC BAND CALCULATION}

Fig. 2 shows calculation models and the coordinate system. The PC slabs have triangular lattice circular airholes. In the SOI PC slab, airholes are only assumed in the Si layer. The PC waveguides lie along the $z$ direction $(\Gamma-\mathrm{J}$ direction of the Brillouin zone). The refractive indexes of $\mathrm{Si}, \mathrm{SiO}_{2}$, and air are assumed to be 3.5, 1.45, and 1.0, respectively. Lattice pitch $a$, airhole diameter $2 r$, and slab thickness $t$ are described by cubic Yee cells, such that digital errors against target sizes are balanced and minimized. Each thickness of the upper and lower claddings is fixed to be $3 t$. The upper and lower boundaries of the analytical space parallel to the $y z$ plane are terminated by the Mur's second-order absorbing boundary condition $(\mathrm{ABC})$ or the Berenger's perfectly matched layer $\mathrm{ABC}$. This allows the investigation of both pure guided modes below the light line and leaky modes in the light cone. For the PC slabs, the lateral four boundaries are terminated by the periodic boundary condition (PBC) expressed by the Floquet theorem with the wavevector $\boldsymbol{k}$ inside the $y z$ plane [17]. Each model includes two unit cells of the PC so that the model is rectangular and the Floquet theorem is easily applied. Each model of the PC waveguides includes 1 and 10 unit cells of the PC in the $z$ and $y$ directions, respectively, and a defect is placed at the center. For them, the lateral two boundaries normal to the line defect are terminated by the PBC. The lateral two boundaries parallel to the line defect are terminated by either the PBC or the ABC. This PBC generates not only waveguide modes confined in the line defect but also spurious slab modes widely spreading in the PC slab and maintained by the periodicity. The ABC allows the elimination of such spurious modes. However, the calculation is sometimes unstable due to the insufficient suppression of reflection by the $A B C$. In this calculation, the $\mathrm{PBC}$ is mainly used, and the $\mathrm{ABC}$ is also used for checking if it is a waveguide mode or a spurious mode. In all calculations, the initial excitation is given at six points with a time series of sinusoidally modulated Gaussian pulses. These points are chosen to be asymmetric with each other in the $y z$ plane and at the center in the $x$ direction. We excite $y$ components of the electric (magnetic) field to investigate TE-like (TM-like) polarized modes in the airbridge PC slab. We expect similar polarized modes in the SOI slab by the same excitation, although the structure is asymmetric and the polarization is not strictly defined. The time step is determined by Courant's condition. After $2^{15}=32768$ time steps, the time series of some field components at some points are Fourier transformed and the peak frequencies are extracted as eigenvalues for the assumed $k$ vector. Finally, photonic bands for each model, which are projected to the $y z$ plane, are obtained by repeating the same calculation but changing $k$. For the PC slabs, we eliminate the extra bands caused by the zone folding for the model with two unit cells. Mode peaks after the Fourier transform are sometimes not clear in the light cone because of the leakage loss. On the other hand, many spurious peaks coming from the periodicity appear for the PC waveguides, as mentioned above.

Fig. 3(a) verifies photonic bands calculated by the FDTD method for TE-like polarization in the airbridge PC slab. Here, 14,8 , and 8 Yee cells are assumed for $a, 2 r$, and $t$, respectively. These parameters were chosen to simulate the exper-

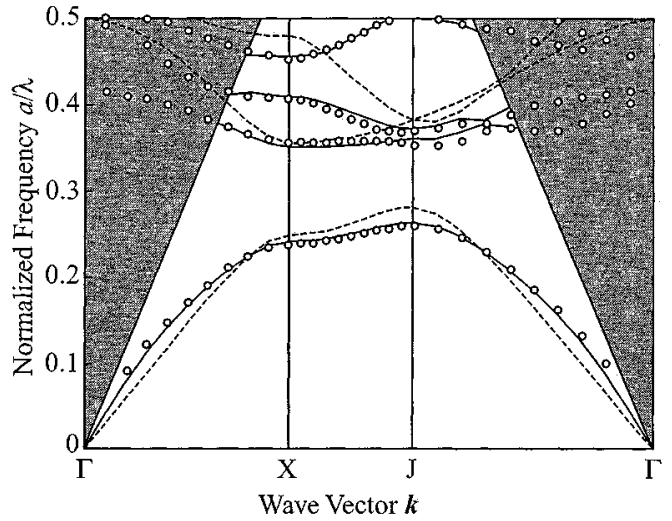

(a)

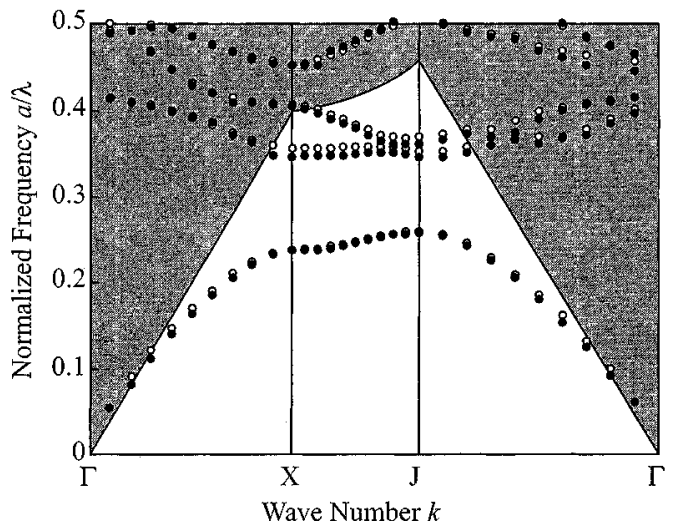

(b)

Fig. 3. Photonic bands calculated for TE-like polarization. (a) Bands for the airbridge PC slab calculated by the FDTD method with 14, 8, and 8 Yee cells for $a, 2 r$, and $t$, respectively (open circles), by the 3-D PWE method with 1475 plane waves, $2 r / a=0.624$ and $t / a=0.571$ (solid curves), and by the 2-D PWE method with 533 plane waves, $2 r / a=0.624$, and an effective slab index of 2.554 (dashed curves). Dark regions denote the air light cone. The lower frequency boundaries of the light cone are air light lines. (b) Bands for the airbridge PC slab (open circles) and the SOI slab (closed circles). Calculation parameters are the same as for (a). Here, the dark region denotes the $\mathrm{SiO}_{2}$ light cone.

imental result shown in Sections IV. However, since the airhole shape described by the Yee cells is not perfectly circular, $2 r / a=8 / 14=0.571$ cannot be considered as the effective diameter. As shown in Fig. 3(a), the FDTD bands almost agree with those calculated by the PWE method by Ho et al. [18] when $2 r / a=0.624$ and $t / a=0.571$ are assumed. The ratio $2 r / a=0.624$ corresponds to the airhole diameter that gives the same airhole area as that assumed in the FDTD calculation. The small discrepancy, as shown in Fig. 3(a), may come from $1 \%$ error in the triangular lattice and/or the deformation of airholes described by the Yee cells in the FDTD calculation. The PBG lies at $a / \lambda=0.27-0.35$. Fig. 3(a) also shows that the range of the PBG can be roughly estimated by the 2-D PWE method with the equivalent slab index 2.55 as the background index. Since the equivalent index is calculated for the airbridge slab at a normalized frequency $a / \lambda=0.27$, error of the 2-D bands against the 3-D bands is increased at higher frequencies. In general, a higher frequency gradually enhances the equivalent index up to the medium index of the slab 3.5, so the 3-D bands are shifted to a lower frequency range. Fig. 3(b) shows that the difference of the FDTD bands for airbridge and SOI PC 


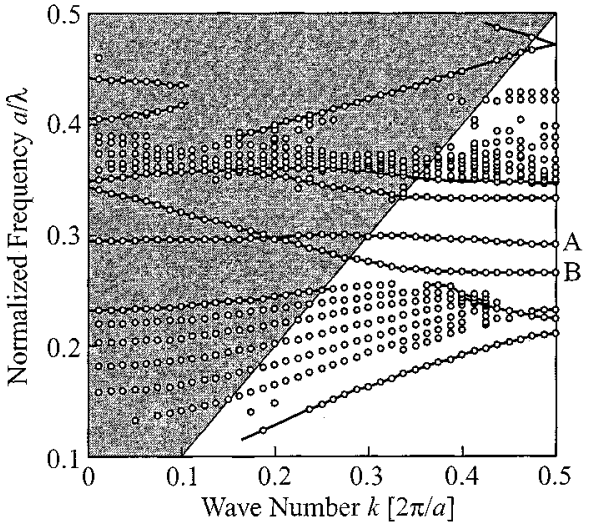

(a)

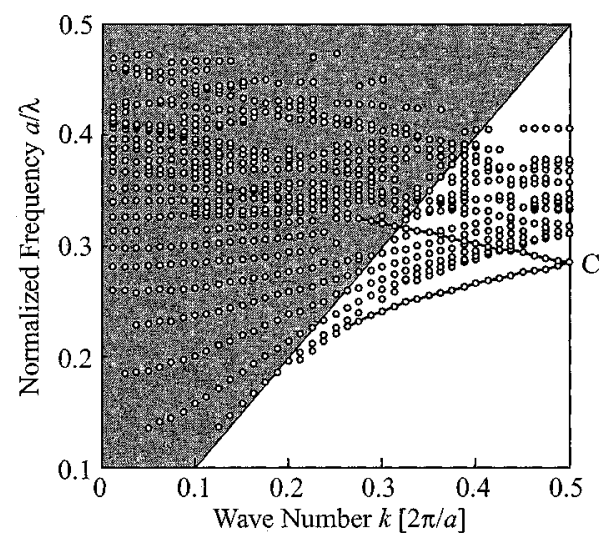

(c)

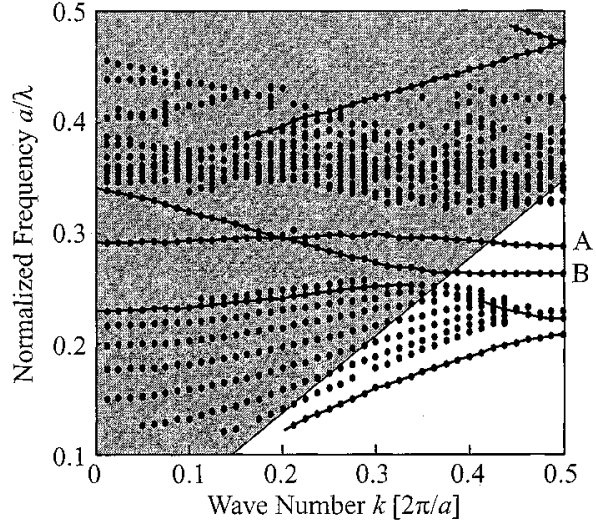

(b)

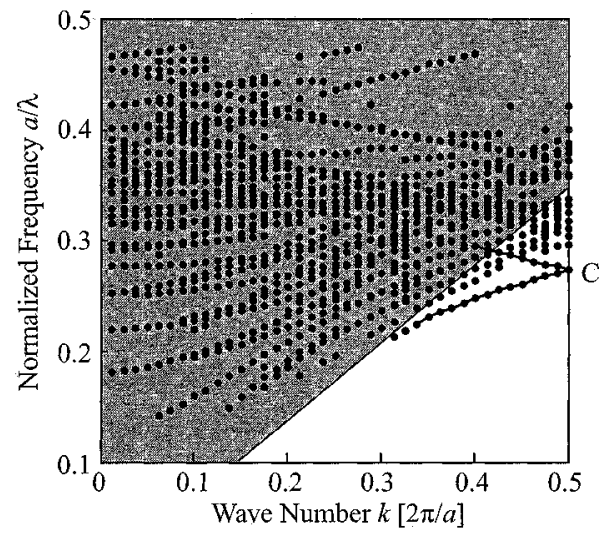

(d)

Fig. 4. Photonic bands calculated for PC waveguides by the FDTD method. The same structural parameters as for Fig. 3 are assumed. (a) and (b) are for TE-like polarization and (c) and (d) for TM-like. (a) and (c) are for the airbridge type waveguide and (b) and (d) for the SOI slab type. Corresponding to this, dark region denotes the air light cone in (a) and (c) and the $\mathrm{SiO}_{2}$ light cone in (b) and (d). A and $\mathrm{B}$ are pure guided mode bands lying in the PBG. C is an index guided mode band.

slabs is small. This indicates that the polarization is almost defined even with the break of symmetry in the SOI slab and the PBG effect is equally expected for these PC slabs.

Fig. 4 shows FDTD bands in the PC waveguides, which are calculated with the $\mathrm{PBC}$ at four lateral boundaries. Here, the same structural parameters as for Fig. 3 are assumed. To distinguish real bands from spurious bands, only those calculated by the FDTD method with the ABC at two lateral boundaries are displayed by solid curves. For TE-like polarization, there are two bands of pure guided modes in the region surrounded by slab mode regions and the light cone, as shown in Fig. 4(a). In general, the PBG is shifted to a higher or lower frequency range by increasing or decreasing the ratio $2 r / a$. The widest PBG is calculated for $2 r / a>0.9$. However, since the light line is simply determined by the cladding index, a wider PBG not affected by the light cone is rather obtained by a smaller airhole. As explained by Notomi et al. [16], two guided modes A and B in Fig. 4(a) originate from the defect mode confined by the PBG and the index guided mode zone folded into the Brillouin zone, respectively. The zone folded band $\mathrm{B}$ is suitable for power transmission because of a large band slope in the PBG, which corresponds to a small group index $n_{g}$ and a large group velocity $c / n_{g}$. This band depends on structural parameters of the waveguide channel, e.g., lattice pitch $a$. Therefore, it is almost fixed against the change of diameter $2 r$. The PBG can be adjusted to this band by employing $2 r / a<0.65$. The effective frequency range of band B below the air light line is $\sim 10 \%$ of the central frequency for $2 r / a \sim 0.55$. This is wide enough to use for fiber communication devices. As mentioned above, Fig. 4 assumes slightly larger airholes. Therefore, in this figure, the effective frequency range is $\sim 5 \%$ of the central frequency and its minimum group index $n_{g}$ is $\sim 4$. This group index is nearly equal to the group index of a rectangular Si channel waveguide [19]. Band A shows an effective frequency range of $2 \%$ and minimum group index $n_{g}$ of $\sim 10$.

Fig. 4(a) and (b) shows that the positions of the waveguide bands in the airbridge type and the SOI slab type are almost the same. The only clear difference we notice is that band peaks for the SOI slab type after the Fourier transform are unclear just above the light line of $\mathrm{SiO}_{2}$. This means that the leakage loss toward the $\mathrm{SiO}_{2}$ cladding is large in this region. However, the frequency range that exhibits a small $n_{g}$ only exists in between the air and $\mathrm{SiO}_{2}$ light lines. In the SOI slab type, bands $\mathrm{A}$ and B show effective frequency ranges of $1 \%$ and $0.5 \%$ and minimum $n_{g}$ of $\sim 10$ and $\sim 24$, respectively. These narrow frequency ranges will make their observation difficult, and the large group index will enhance the practical propagation loss. Fig. 4(c) and (d) shows FDTD bands for TM-like polarization. Similar to the discussion by Lončar et al. [15], there are no PBGs, but an index guided mode exists below the slab mode region. This mode lies 
(1) SOI wafer

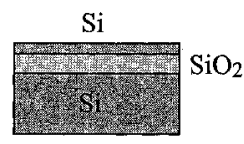

(4) Transcription

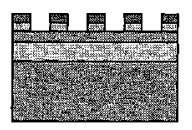

(2) Evaporation

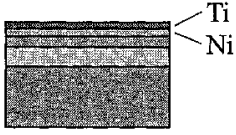

(5) ICP etching

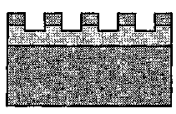

(SOI slab)
(3) EB lithography

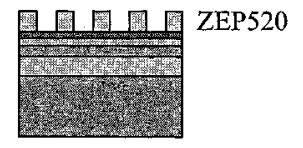

(6) HF etching

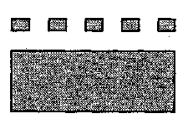

(Airbridge)
Fig. 5. Fabrication process of a PC waveguide.

in the frequency range almost overlapping with the effective frequency range for TE-like polarization.

\section{FABRICATION}

We prepared a unibond type SOI wafer (SOI TEC Inc.) having an $\mathrm{SiO}_{2}$ intermediate layer $1.0 \mu \mathrm{m}$ thick and a top $p$-type $\mathrm{Si}$ layer $0.30-0.32 \mu \mathrm{m}$ thick. The Si layer is slightly thicker than the single-mode condition as the slab waveguide at $\lambda=1.55$ $\mu \mathrm{m}$. However, by the perturbation theory described in Section V, the higher order mode suffers nearly three times greater scattering loss than that for the fundamental mode in the slab with surface roughness, since the field penetration of this mode into air is much larger than that of the fundamental mode. Therefore, we can expect quasi-single-mode behavior in this slab. Besides, the slab mode calculation by the interference matrix method [20] indicates that the $\mathrm{SiO}_{2}$ layer is thick enough to reduce the leakage loss toward the $\mathrm{Si}$ substrate to less than $10^{-4}$ $\mathrm{dB} / \mathrm{mm}$. Since the doping concentration of the Si layer is of the order of $10^{16} \mathrm{~cm}^{-3}$, the free carrier absorption loss of this layer may be negligible compared with the propagation loss described in Section IV. Also, the interband absorption loss is negligible at target wavelengths of this study, i.e., $\lambda=1.36$ to $1.60 \mu \mathrm{m}$, even though Si has a decaying tail of absorption spectrum toward wavelengths longer than the electronic band edge due to the indirect transition.

The fabrication process is outlined in Fig. 5. On a cleaved piece of the SOI wafer, $\mathrm{Ni}$ and $\mathrm{Ti}$ are evaporated as etching masks. Then, a PC waveguide pattern is drawn by electron beam (EB) lithography. We used an EB machine (ELS 3700, Elionix Co. Ltd.) with a $\mathrm{LaB}_{6}$ filament, and positive resist ZEP 520 (Zeon Corp.). The pattern includes various PC's with $a=0.38$ to $0.46 \mu \mathrm{m}$ and $2 r=0.14$ to $0.24 \mu \mathrm{m}$, and different length waveguide channels. This pattern is transferred to Ti using $\mathrm{CF}_{4}$ reactive ion etching and to $\mathrm{Ni}$ using $\mathrm{Ar}$ ion milling. Finally, the $\mathrm{Si}$ layer is etched by the inductively coupled plasma (ICP) etching. We used the ICP machine RIE-200ip (Samco International Inc.) with $\mathrm{CF}_{4}$ and $\mathrm{Xe}$ gases. The top Ti mask is removed during the ICP etching. The Ni mask is removed by $\mathrm{HCl}$ etching for five minutes to several hours. This etching time is changed by the amount of the deposition of a fluoride on the Ni mask during the ICP etching. The $\mathrm{SiO}_{2}$ layer is removed by additional HF etching for the airbridge, while this etching is omitted for the SOI PC slab.
As shown in Fig. 6, the fabricated PC has some imperfections. The sidewalls of airholes are almost vertical with $<1^{\circ}$ angle error. The top surface of the Si layer has a $10-20 \mathrm{~nm}$ roughness. This seems to be caused by the fluoride deposition and the shallow transcription of its irregular pattern to the $\mathrm{Si}$ layer during the $\mathrm{HCl}$ etching. When the $\mathrm{HCl}$ etching is long, the Si layer is slightly thinned by the repetition of the natural oxidation and its removal. The final thickness of this sample is $250-300 \mathrm{~nm}$. Each airhole is elliptical with the maximum oblateness of $9 \%$. An edge roughness of airholes is from \pm 10 to $\pm 15 \mathrm{~nm}$. This leads to a fluctuation in the average diameter of airholes of maximally $\pm 4 \%$. In this experiment, the EB CAD data was constructed by a 10 -nm grid. It causes $1 \%$ error in the triangular lattice theoretically, but it looks negligible in the formed pattern. Besides, the ICP etching typically reaches deeper by $\sim 0.3 \mu \mathrm{m}$ from the $\mathrm{Si} / \mathrm{SiO}_{2}$ interface. Ideally, this etching should be stopped at the interface to demonstrate the theoretical model of the SOI slab type, but the automatic stop is difficult for the etching condition used.

\section{MeAsurement}

In general, the fabrication of a PC waveguide of millimeter order is difficult because of the limited number of grid points in the EB CAD data. In our system, for example, the total number of grid is $20000 \times 20000$. Therefore, the length of a straight waveguide is limited to $200 \mu \mathrm{m}$ against a $10-\mathrm{nm}$ grid without using a high-accuracy stage system. The insertion and extraction of light are also difficult because of the submicrometer cross-sectional core and the short waveguide. To simplify the loss estimation, we previously employed the Fabry-Perot resonance method [10]. In this method, the propagation loss is estimated from the finesse of the resonance inside the waveguide with input and output end facets. This method is advantageous, since the estimation is nondestructive and independent of light inserting and extracting conditions. For PC waveguides, however, this method has an uncertainty coming from the facet reflectivity, which can be changed by the phase of the PC pattern at cleaved facets. Another method usually used is the cut back method of the waveguide. However, it is not easy to cleave a waveguide shorter than $200 \mu \mathrm{m}$ into several pieces with the same facet condition. The butt joint of a PC waveguide and index confinement waveguides simplifies this cleavage. However, the joint loss introduces another uncertainty in the estimation.

To avoid these difficulties and uncertainties, we used a method described below. The optical setup is shown in Fig. 7. Two tunable laser sources continuously cover $\lambda=$ $1.36-1.60 \mu \mathrm{m}$. TE or TM polarization is selected by polarizers and wave plates. The light is focused to a cleaved end of a PC waveguide with a $\sim 1-\mu \mathrm{m}$ spot diameter by two objective lenses. One sample piece includes four waveguides with different lengths. Each waveguide has a large square hole at the other end. The light output is detected by a vidicon camera with a microscope system from the top of the waveguide. As shown in Fig. 8, light radiated from PC waveguides is expected to have a wide radiation angle over $180^{\circ}$ due to the waveguide spotsize being smaller than the diffraction limit in air and 


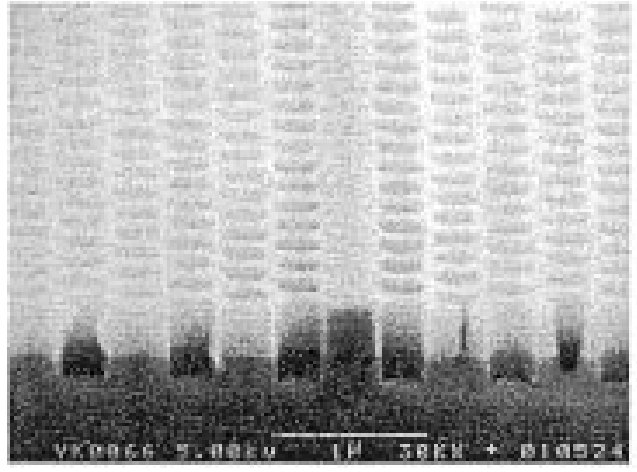

(a)

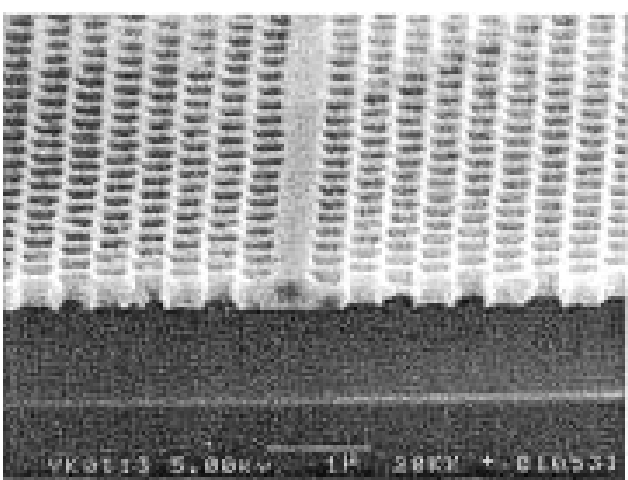

(c)

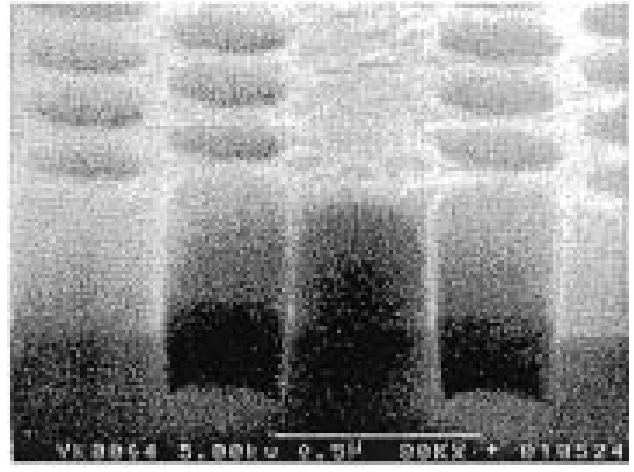

(b)

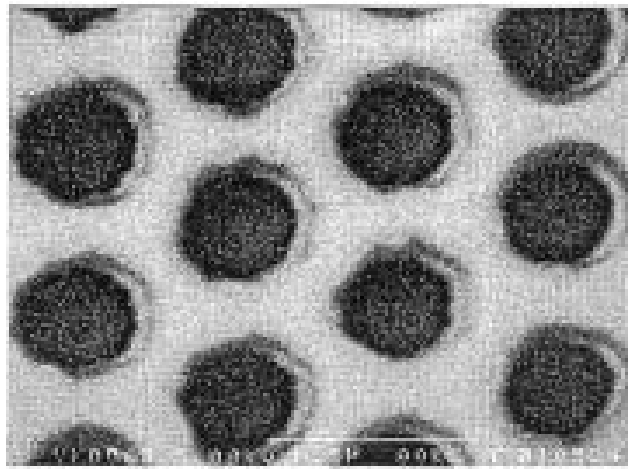

(d)

Fig. 6. Photographs of PC waveguides observed by a field emission type scanning electron microscope. (a) SOI slab type PC waveguide, (b) magnified view, (c) airbridge type PC waveguide, and (d) top view of the PC.

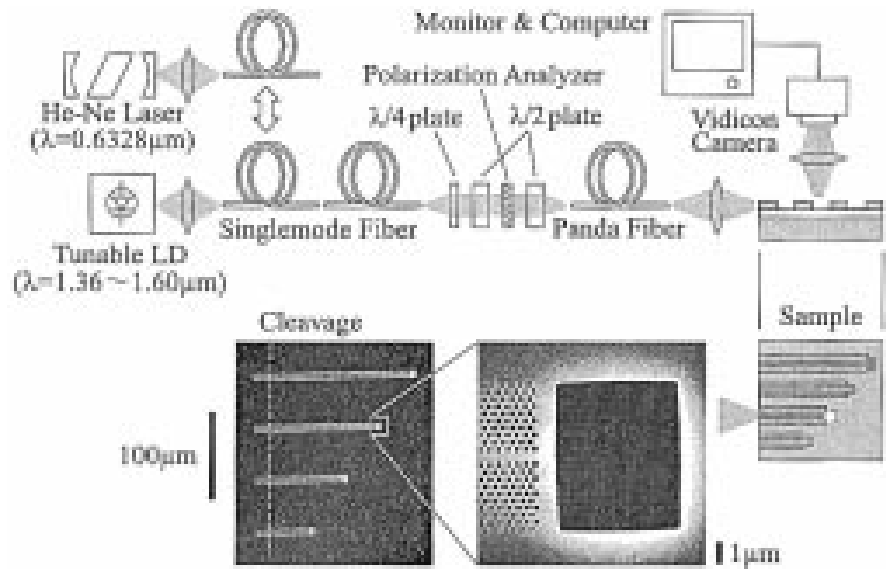

Fig. 7. Optical setup for the observation of light propagation in PC waveguides and top view of a sample including four different length PC waveguides with square holes.

due to the reflection at the end facet. Therefore, meaningful light power can be detected from the top even without any irregular scattering at the facet. This detection is also effective for eliminating $\mathrm{SiO}_{2}$ cladding modes. Relative output intensity from the waveguide is evaluated by compensating the intensity on the vidicon camera with a measured nonlinearity factor of 0.7 .

Fig. 9 shows the observed near-field patterns (NFPs) of light propagation in an airbridge type waveguide. In this sample, $a=$ $0.42 \mu \mathrm{m}, 2 r=0.24 \pm 0.01 \mu \mathrm{m}$, and $t=0.28 \pm 0.02 \mu \mathrm{m}$. The length of the waveguide for TE-like polarization is $85 \mu \mathrm{m}$ and $42 \mu \mathrm{m}$ for TM-like polarization. As shown in Fig. 9(a) for

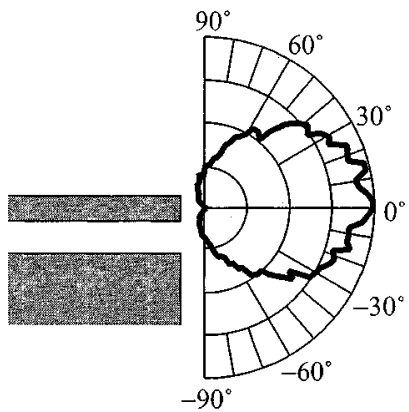

(a)

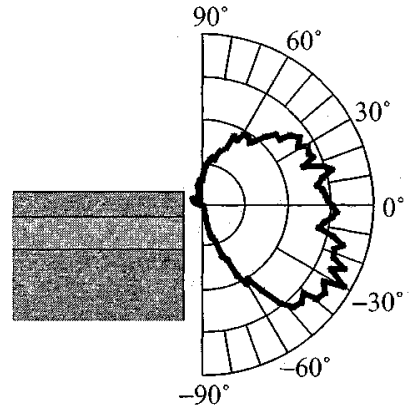

(b)
Fig. 8. Calculated far field patterns of TE guided mode radiated from unpatterned slabs at $\lambda=1.55 \mu \mathrm{m}$. The 2-D FDTD method is used. Thickness $t$ is assumed to be $0.32 \mu \mathrm{m}$. (a) Airbridge slab and (b) SOI slab.

TE-like polarization, the light spot at the output end is clear at $\lambda=1.38-1.39 \mu \mathrm{m}$ and $1.41-1.55 \mu \mathrm{m}$. In this figure, light scattering along the waveguide, as we reported for three line defect SOI slab type waveguides [10], is not observed at all. This is the evidence of pure guided modes not affected by the light cone. The propagation loss spectrum is evaluated by comparing light outputs from the different length waveguides, as shown in Fig. 10. To avoid the influence of the chromatic aberration of the objective lenses in the above wavelength range, we adjust the setup to maintain the optimum coupling condition for each wavelength. However, this estimate includes a roughly $\pm 20 \%$ error. The fluctuation of the brightness and the nonlinearity in the video flame of the vidicon camera is rather small. The error is caused mainly by the scattered data of the output intensity for the different length waveguides, which are caused by the very 


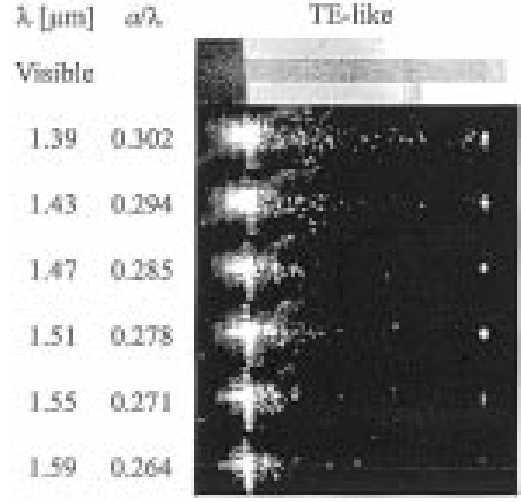

(a)

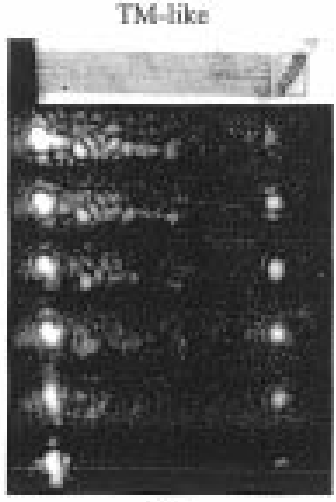

(b)
Fig. 9. NFPs of light propagation in an airbridge type $\mathrm{PC}$ waveguide, which are observed against various wavelengths from the top. $a=0.42 \mu \mathrm{m}, 2 r=$ $0.24 \pm 0.01 \mu \mathrm{m}$ and $t=0.28 \pm 0.02 \mu \mathrm{m}$. The waveguide length of the sample for (a) TE-like polarization is $85 \mu \mathrm{m}$ and that for (b) TM-like polarization $42 \mu \mathrm{m}$.

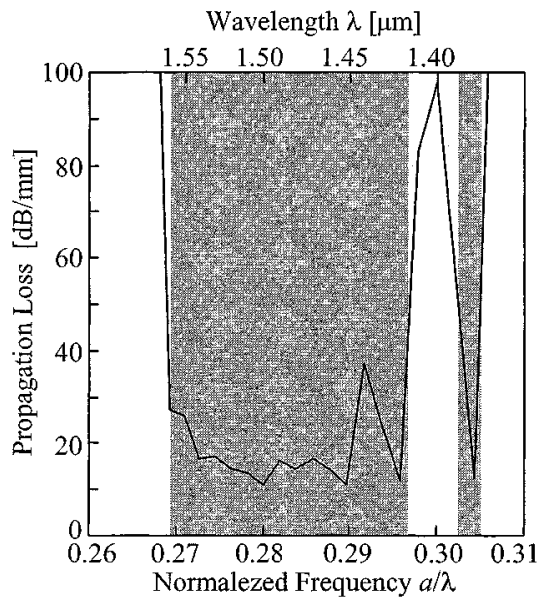

Fig. 10. Propagation loss spectrum for TE-like polarization in an airbridge type PC waveguide. The sample is the same as that in Fig. 9.

severe light coupling condition and the structural imperfection of each waveguide. From Fig. 10, the minimum loss evaluated is $11 \mathrm{~dB} / \mathrm{mm}$. Considering the irregular scattering at PC claddings as the origin of the propagation loss, the loss coefficient $\alpha$ is expressed as [20]

$$
\alpha=C(1-R) \tan \theta / w \approx C(1-R) /\left\lfloor w \sqrt{1+(2 n w / \lambda)^{2}}\right\rfloor
$$

where $C$ is a constant value, $R$ is the reflectivity of PC claddings, $\theta$ is the ray angle of the guided mode measured from the $z$ axis, $w$ is the modal width, and $n$ is the core index. For $n=3, w>0.5 \mu \mathrm{m}$, and $\lambda=1.5 \mu \mathrm{m},(2 n w / \lambda)^{2} \gg 1$, and $\alpha$ is almost proportional to $(1-R) / w^{2}$. Assuming that $R$ has an angular dependence similar to that of the Fresnel reflection [21] and that $w$ is proportional to the channel width, $\alpha=11 \mathrm{~dB} / \mathrm{mm}$ for the single-line-defect PC waveguide suggests the possibility of $\alpha<1 \mathrm{~dB} / \mathrm{mm}$ in the three-line-defect waveguide. This means a much lower loss in this work than in previous works [10], [11]. The two wavelength ranges exhibiting low propagation loss are explained by FDTD bands A and B of the guided modes, as shown in Fig. 11(a). The 3\%-7\% error between theoretical and experimental results may come from the digital error in the FDTD calculation. Fig. 12 shows simulated electric

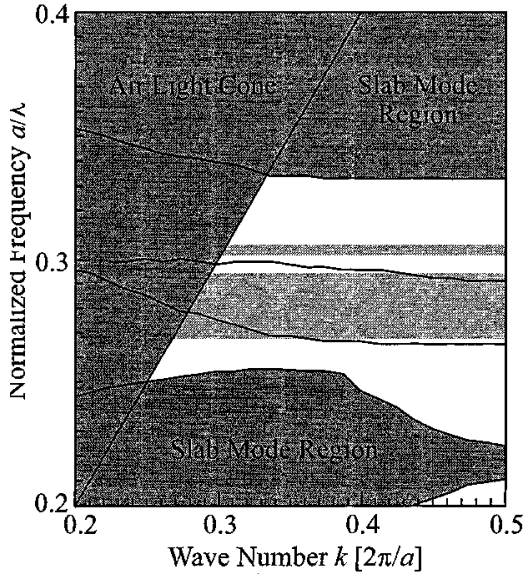

(a)

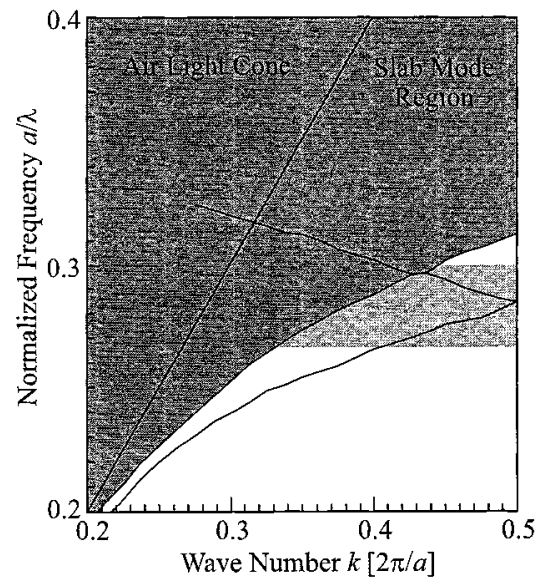

(b)

Fig. 11. Photonic bands for (a) TE-like and (b) TM-like polarizations in an airbridge type PC waveguide calculated, as shown in Fig. 3. The bright gray regions indicate frequency ranges that showed low loss propagation in the experiment.
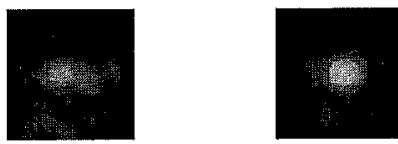

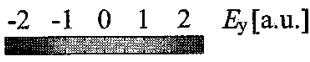

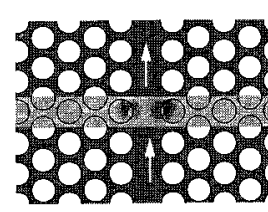

(a)
Fig. 12. Simulated profiles of the $y$ component of the electric field and observed NFP's for TE-like polarization in the airbridge type PC waveguide. For the calculation, the same parameters as for Fig. 3 and $k=0.8 \pi / a$ are assumed. (a) Profile of mode A and observed NFP at $\lambda=1.39 \mu \mathrm{m}$. (b) Profile of mode B and observed NFP at $\lambda=1.51 \mu \mathrm{m}$. (c) Profile of a slab mode.

field profiles for these guided modes and a slab mode. Fig. 12 also shows magnified NFP's for the corresponding frequency ranges. Although their size is of submicron order and cannot be fully resolved by the microscope system, the single and double lobe profile are observed. They also correspond to simulated profiles of even and odd modes. One difference of the experimental result from the theoretical expectation is that the minimum propagation loss for band $\mathrm{A}$ is the same as that for 


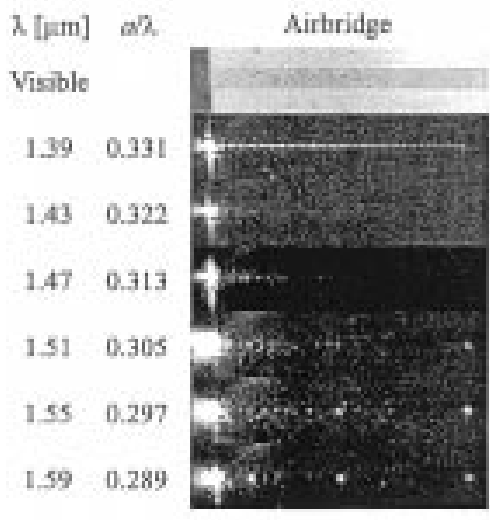

(a)

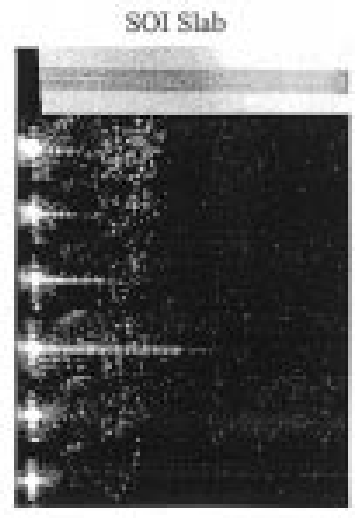

(b)
Fig. 13. NFPs of light propagation for TE-like polarization in PC waveguides, which are observed against various wavelengths from the top. $a=0.46 \mu \mathrm{m}$, $2 r=0.23 \pm 0.01 \mu \mathrm{m}$ and $t=0.30 \pm 0.02 \mu \mathrm{m}$. The waveguide length is $106 \mu \mathrm{m}$. (a) Airbridge type (after HF etching) and (b) SOI slab type (before HF etching of the same sample).

band B. As shown in Section II, the group index of band A is 2.5 times that of band $\mathrm{B}$, so we expect a smaller group velocity and larger loss for band A. It is not simple to explain this, since the scattering loss by the irregularity is related to the coupling efficiency between the irregularity and the mode field, both of which are complex in the photonic crystal.

Fig. 9(b) shows NFPs for TM-like polarization. The light propagation is also observed at $\lambda=1.40-1.57 \mu \mathrm{m}$. This almost agrees with FDTD bands, as shown in Fig. 11(b). The NFP shows a single lobe profile, which can be explained by the calculated index guided mode. Fig. 13 compares NFPs for TE-like polarization in airbridge type and SOI slab type waveguides. Compared with NFPs in Fig. 9(a), efficient light propagation in the airbridge type is observed at a lower frequency range due to the larger lattice pitch and slightly thicker slab of this sample. At $\lambda=1.40-1.48 \mu \mathrm{m}$, no light propagation is observed due to the influence of the air light line. At $\lambda<1.40 \mu \mathrm{m}$, light propagation with scattering along the waveguide is observed, which shows the existence of a relatively low loss leaky mode in the light cone. On the other hand, both light propagation and scattering along the waveguide are not clear in the SOI slab type. This shows that the $\mathrm{SiO}_{2}$ light cone strongly affects the light propagation, and that bands below the light line cannot guide a meaningful light power practically due to the narrow effective frequency range and/or the large group index. The frequency $a / \lambda=0.305$ is just on the air light line condition. This is the reason for the relatively strong light scattering.

\section{DiscusSiOn ON SCATTERING LosS}

Since pure guided modes do not suffer any theoretical losses, the origin of the observed propagation loss is the scattering loss by various imperfections in fabricated waveguides, as shown in Section III. It is not simple to estimate the scattering loss by each imperfection. However, the deformation of the triangular lattice and the extension of airholes into $\mathrm{SiO}_{2}$ will not change photonic bands of the guide modes so much, although they modify or inhibit the rigorous discussion of pure guided modes and leaky

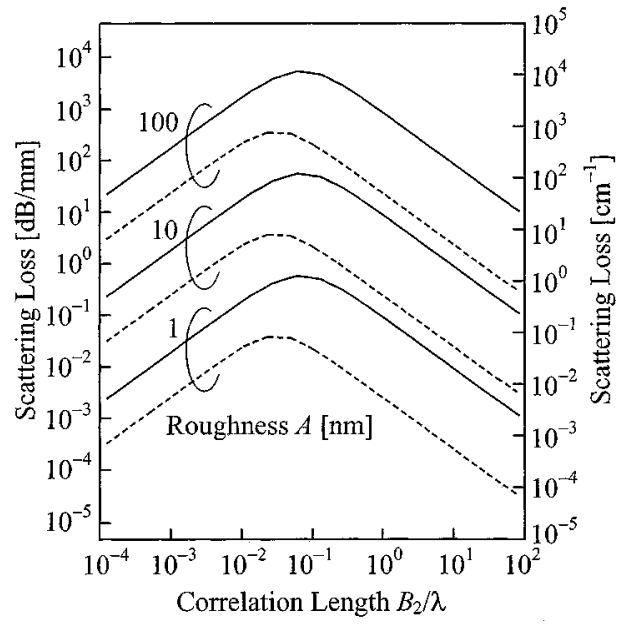

Fig. 14. Scattering loss calculated for an index confinement slab waveguide by the perturbation theory. Wavelength $\lambda$ is $1.55 \mu \mathrm{m}$. Indexes of core and claddings are 3.02 and 1.0, respectively. The thiickness of the core satisfies the cutoff condition of the first higher order mode. The correlation length between boundaries $B_{1}$ is assumed to be $0.01 \lambda$. Solid curves indicate the total scattering loss against various roughness amplitude $A$, and dotted curves indicate the scattering loss caused by the coupling of guided mode with the backward guided mode.

modes separated by the light line. We consider that the main origins of the scattering loss are the surface and sidewall roughness and the fluctuation in airhole diameter.

The scattering loss in such a structure occurs by the coupling of the guided mode with radiation modes and with the backward guided mode. This loss is calculated for an index confinement waveguide by perturbation theory [22], as shown in Fig. 14. Here, a symmetric slab waveguide with a core index $n_{1}$ of 3.02 , a cladding index $n_{2}$ of 1.0 and the cutoff thickness of the higher order mode at $\lambda=1.55 \mu \mathrm{m}$ are assumed. The irregularity is expressed by the fluctuation of boundaries with roughness amplitude $A$ and correlation lengths $B_{1}$ between the boundaries and $B_{2}$ inside each boundary. The exponentially decaying function is assumed for these correlation lengths. In general, the scattering loss is proportional to $A^{2}$ and nearly proportional to $\Delta^{2.5}$ under the single-mode condition [23], where $\Delta$ is the relative index difference defined as $\left(n_{1}^{2}-n_{2}^{2}\right) / 2 n_{1}^{2}$. Therefore, the scattering loss is much larger in such a high- $\Delta$ waveguide than in a low- $\Delta$ waveguide such as silica-based waveguides. We fabricated an index confinement single-mode waveguide with a $\mathrm{Si}$ rectangular core into an SOI wafer and measured the propagation loss to be $\sim 10 \mathrm{~dB} / \mathrm{mm}$ at $\lambda=1.55 \mu \mathrm{m}$ [19]. This is a reasonable value against a roughness $A \sim 10 \mathrm{~nm}$ and a correlation length $B_{2} \sim 0.01 \lambda$, both of which were measured for this waveguide. The roughness will be reduced and the correlation length will be controlled to some extent by the optimization of the fabrication process. However, it seems difficult to drastically reduce the propagation loss to less than $0.01 \mathrm{~dB} / \mathrm{mm}$ (a normal value for silica waveguides), since a very small roughness of an atomic layer order is required for this value.

One expectation for the PC waveguide is that the PBG inhibits radiation modes and essentially reduces the scattering loss. If radiation modes are absolutely inhibited, the scattering loss in the index confinement waveguide will be reduced 1-2 orders of magnitude, as shown by dashed curves in Fig. 14. Of course, the 
scattering loss in PC waveguides may be different due to the different mode profile and the existence of the light cone. However, since irregularities are mainly introduced into the sidewalls of the airholes during the etching process, the main origin of the propagation loss should not be the scattering into the light cone but the in-plane scattering, which can be suppressed by PBG.

\section{CONCLUSION}

Single-line-defect optical waveguides in PC slabs were fabricated into an SOI wafer. A clear light propagation was not observed in the SOI slab type waveguide but observed in the airbridge type. Wavelength ranges showing efficient light propagation for the incidence of TE polarization were well explained by photonic bands lying in the PBG, which are calculated by the 3-D FDTD method. The light propagation by the index confinement mode was also observed for the incidence of TM polarization at the same frequency range. The minimum propagation loss for TE-like polarization was estimated to be $11 \mathrm{~dB} / \mathrm{mm}$ by comparing the light output from different length waveguides. This value is almost comparable to that in an index confinement waveguide with a rectangular $\mathrm{Si}$ core, even though the PC waveguide has various and larger irregularities. This result and a calculation of scattering loss by perturbation theory suggest that the propagation loss limited by in-plane light scattering can be 1-2 orders of magnitude lower than in the index waveguide. If this expectation is correct, the use of a PC waveguide is advantageous not only at bends, branches, etc., but also at straight parts in a photonic chip.

\section{ACKNOWLEDGMENT}

The authors would like to thank Prof. Y. Kokubun, Yokohama National University, and Prof. K. Iga and Prof. F. Koyama, Tokyo Institute of Technology, for encouragement and helpful suggestions.

\section{REFERENCES}

[1] R. D. Meade, A. Devenyi, J. D. Joannopoulos, O. L. Alerhand, D. A. Smith, and K. Kash, "Novel applications of photonic band gap materials: Low-loss bends and high $Q$ cavities," Appl. Phys. Lett., vol. 75, pp. $4753-4755,1994$.

[2] A. Mekis, J. C. Chen, I. Kurand, S. Fan, P. R. Villeneuve, and J. D. Joannopoulos, "High transmission through sharp bends in photonic crystal waveguides," Phys. Rev. Lett., vol. 77, pp. 3787-3790, 1996.

[3] J. Yonekura, M. Ikeda, and T. Baba, "Analysis of finite 2-D photonic crystals of columns and lightwave devices using the scattering matrix method," J. Lightwave Technol., vol. 17, pp. 1500-1508, 1999.

[4] J. S. Foresi, P. R. Villeneuve, J. Ferrera, E. R. Thoen, G. Steinmeyer, S. Fan, J. D. Joannopoulos, L. C. Kimerling, H. I. Smith, and E. P. Ippen, "Photonic bandgap microcavities in optical waveguides," Nature, pp. 143-145, 1997.

[5] T. Baba, N. Fukaya, and J. Yonekura, "Observation of light propagation in photonic crystal optical waveguides with bends," Electron. Lett., vol. 35, pp. 654-655, 1999.

[6] N. Fukaya, D. Ohsaki, and T. Baba, "2-D photonic crystal waveguides with 60-degree-bends in a thin slab structure," Jpn. J. Appl. Phys., vol. 39, pp. 2619-2623, 2000.

[7] S. Y. Lin, E. Chow, S. G. Johnson, and J. D. Joannopoulos, "Demonstration of highly efficient waveguiding in a photonic crystal slab at the 1.5- $\mu$ m wavelength," Opt. Lett., vol. 25, pp. 1297-1299, 2000.
[8] M. Tokushima, H. Kosaka, A. Tomita, and H. Yamada, "Lightwave propagation through a 120 degrees sharply bent single-line-defect photonic crystal waveguide," Appl. Phys. Lett., vol. 76, pp. 952-954, 2000.

[9] M. Lončar, D. Nedeljković, T. Doll, J. Vučković, A. Scherer, and T. P. Pearsall, "Waveguiding in planar photonic crystals," Appl. Phys. Lett., vol. 77, pp. 1937-1939, 2000.

[10] T. Baba and N. Fukaya, "Light propagation characteristics of defect waveguides in a photonic crystal slab," in Photonic Crystals and Light Localization, M. Soukoulis, Ed. Boston, MA: Kluwer Academic, 2001, pp. 105-116.

[11] C. J. Smith, H. Benisty, S. Olivier, M. Rattier, C. Weisbuch, T. F. Krauss, R. M. De La Rue, R. Houdré, and U. Oesterle, "Low-loss channel waveguides with two-dimensional photonic crystal boundaries," Appl. Phys. Lett, vol. 77, pp. 2813-2815, 2000.

[12] S. G. Johnson, S. Fan, P. R. Villeneuve, and J. D. Joannopoulos, "Guided modes in photonic crystal slabs," Phys. Rev. B, vol. 60, pp. 5751-5758, 1999.

[13] A. Chutinan and S. Noda, "Waveguides and waveguide bends in two-dimensional photonic crystal slabs," Phys. Rev. B, vol. 62, pp. 4488-4492, 2000 .

[14] S. G. Johnson, P. R. Villeneuve, S. Fan, and J. D. Joannopoulos, "Linear waveguides in photonic crystal slabs," Phys. Rev. B, vol. 62, pp. 8212-8222, 2000

[15] M. Lončar, T. Doll, J. Vučković, and A. Scherer, "Design and fabrication of silicon photonic crystal optical waveguides," J. Lightwave Technol., vol. 18, pp. 1402-1411, 2000.

[16] M. Notomi, A. Shinya, K. Yamada, J. Takahashi, and I. Yokohama, "Single-mode transmission within photonic bandgap of width-varied single-line-defect photonic crystal waveguides on SOI substrates," Electron. Lett., vol. 37, pp. 293-295, 2001.

[17] C. T. Chan, Q. L. Yu, and K. M. Ho, "Order- $N$ spectral method for electromagnetic waves," Phys. Rev. B, vol. 51, pp. 16 635-16 642, 1995.

[18] K. M. Ho, C. T. Chan, and C. M. Soukoulis, "Existence of a photonic band gap in periodic structures," Phys. Rev. Lett., vol. 65, pp. $3152-3155,1990$

[19] A. Sakai, G. Hara, and T. Baba, "Propagation characteristics of ultra-high $\Delta$ optical waveguide on silicon-on-insulator substrate," Jpn. J. Appl. Phys., vol. 40, pp. L383-L385, 2001.

[20] T. Baba, Y. Kokubun, T. Sakaki, and K. Iga, "Loss reduction of an ARROW waveguide in shorter wavelength and its stack configuration," J. Lightwave Technol., vol. 6, pp. 1440-1445, 1988.

[21] T. Baba and Y. Kokubun, "High efficiency light coupling from antiresonant reflecting optical waveguide to integrated photodetector using antireflecting layer," Appl. Opt., vol. 29, pp. 2781-2792, 1990.

[22] Y. Suematsu and K. Furuya, "Propagation mode and scattering loss of a two-dimensional dielectric waveguide with gradual distribution of refractive index," IEEE Trans. Microwave Theory Tech., vol. MTT-20, pp. 524-531, 1972.

[23] Y. Suematsu and K. Furuya, "Scattering loss of a thin slab waveguide" (in Japanese), Appl. Phys. (Oyobutsuri), vol. 42, pp. 938-942, 1973.
Toshihiko Baba (M'93) was born in 1962. He received the B.E., M.E., and $\mathrm{Ph} . \mathrm{D}$. degrees, all from the Division of Electrical and Computer Engineering, Yokohama National University, Japan, in 1985, 1987, and 1990, respectively.

During his Ph.D. work, he was engaged in antiresonant reflecting optical waveguides and integrated lightwave circuits. In 1990, he joined the Precision and Intelligence Laboratory, Tokyo Institute of Technology as a Research Associate and began research on vertical-cavity surface-emitting lasers (VCSELs). In 1991, he reported the first calculation of spontaneous emission factor in VCSELs. In 1993, he achieved the first RT CW operation of a long-wavelength VCSEL. In 1994, he became an Associate Professor at Yokohama National University. He had also held a Guest Associate Professor at Tokyo Institute of Technology from 1994 to 1998. His current interests are microcavity lasers, photonic crystals, spontaneous emission control, micro-electro-mechanical systems, nonlinear optics and quantum computing.

Dr. Baba is a member of the Institute of Electronics, Information and Communication Engineers of Japan (IEICE), the Japan Society of Applied Physics, and the American Physical Society. He received the Niwa Memorial Prize in 1991, the Best Paper Award of Microoptic Conference in 1993 and 1999, the Paper Award and the Academic Encouragement Award from the IEICE in 1994, and Marubun Research Encouragement Award in 2000. 
Ayumu Motegi was born in 1979. He received the B.E. degree from the Division of Electrical and Computer Engineering, Yokohama National University, Japan,

in 2001. He is currently working toward the M.E. degree at the same university.

His studies concern the photonic crystal line-defect optical waveguide.

Mr. Motegi is a member of the Japan Society of Applied Physics.

Takeshi Iwai was born in 1978. He received the B.E. degree and the M.E. degree from the Department of Electrical and Computer Engineering, Yokohama National University, Japan, in 2000 and 2002, respectively.

He is currently with NEC Corporation, Tokyo, Japan. His research concerns the design of photonic crystal line-defect waveguides and their application to functional nonlinear devices.

Mr. Iwai is a member of the Japan Society of Applied Physics.

Naoyuki Fukaya was born in 1976. He received the B.E. and M.E. degrees from the Department of Electrical and Computer Engineering, Yokohama National University, Japan, in 1999 and 2001, respectively.

During his thesis work, he studied the line-defect waveguide in the photonic crystal slab and succeeded in the first observation of light propagation in this type of waveguide in 1998. He is currently with Furukawa Electric Inc., Yokohama Laboratories, Yokohama, Japan, working on laser diodes for fiber communications.

Mr. Fukaya is a member of the Japan Society of Applied Physics. He received the best paper award from Micro-Optics Conference in 1999.
Yoshitaka Watanabe was born in 1977. He received the B.E. degree from the Department of Electrical and Computer Engineering, Yokohama National University, Japan, in 2001.

He is currently with Toyota, Inc., Aichi, Japan. During his undergraduate work, he studied the photonic band calculation by the finite difference time domain method.

Atsushi Sakai was born in 1970. He received the B.E., M.E. and Ph.D. degrees from the Department of Electrical and Computer Engineering, Yokohama National University, Japan, in 1997, 1999, and 2002, respectively.

He is currently with Ricoh Company, Ltd., Yokohama, Japan. His primary research concerns the numerical analysis of various micro-photonic devices by the finite difference time-domain method and the development of their fabrication process and evaluation method. His work also covers near-field optics in which a microdisk laser is directly used as a probe head. His recent research interests also include the Si photonic wire waveguide on SOI wafer for ultra-high density optical wiring.

Dr. Sakai is a member of the Japan Society of Applied Physics. 